\title{
LAS COMPETENCIAS TIC EN LOS \\ ESTUDIANTES UNIVERSITARIOS DE PRIMER INGRESO
}

\author{
ICT SKILLS IN FIRST-YEAR UNIVERSITY STUDENTS
}

\section{RESUMEN}

El continuo desarrollo de la Sociedad del Conocimiento obliga a que las instituciones de educación superior deban preparar a sus profesionales con cada vez mayores competencias en el uso de las TIC aplicadas a diferentes campos de las Ciencias. En este sentido, y para poder adaptar los objetivos de aprendizaje de las asignaturas relacionas con las TIC a las reales necesidades de los estudiantes, es fundamental contar con un diagnóstico inicial de estas competencias de los estudiantes cuando llegan a la universidad. Así, el objetivo del presente trabajo fue investigar las percepciones que tienen los estudiantes que ingresan a la Facultad de Ciencias de la Educación, de la Universidad Nacional de Chimborazo en Ecuador, sobre el manejo de sus competencias TIC. Para esto se diseñó un estudio no experimental de alcance descriptivo, aplicando la técnica de la encuesta con un instrumento previamente diseñado y validado en la Universidad Metropolitana de Ciencias de la Educación de Chile. Los resultados muestran ciertas preferencias de uso de algunas herramientas y recursos web 2.0 tanto para trabajo colaborativo, almacenamiento en la nube, para tomar notas, entre otras. Así como, su auto-percepción sobre el manejo de algunas herramientas tecnológicas.

Palabras clave: TIC; competencias digitales; educación universitaria; estudiantes universitarios

\section{ABSTRACT}

The continuous development of the Society of Knowledge requires higher education institutions to prepare their professionals with increasing skills in the use of ICT applied to different fields of science. In this sense, and to adapt the learning objectives of the subjects related to ICT to the real needs of the students, it is essential to have an initial diagnosis of these students' competences when they get into the university. Therefore, the objective of this paper was to investigate the perceptions of students who enter the Faculty of Educational Sciences of the National University of Chimborazo in Ecuador, on the management of their ICT skills. For this purpose, a non-experimental study of descriptive scope was designed, applying the survey technique with a previously developed and validated instrument on the Metropolitan University of Education Sciences of Chile. The results show certain preferences for the use of some tools and web 2.0 resources for collaborative work, cloud storage, note-taking, among others, as well as their self-perception about the use of some technological tools.

Keywords: ICT; digital skills; university education; university students

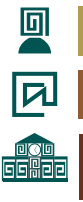

Patricio Humanante-Ramos

phumanante@unach.edu.ec

Universidad Nacional de Chimborazo.

\section{Ma. Eugenia Solís-Mazón}

mesolis@unach.edu.ec

Universidad Nacional de Chimborazo.

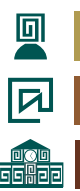

Jorge Silva-Castillo

jsilva@unach.edu.ec

Universidad Nacional de Chimborazo.

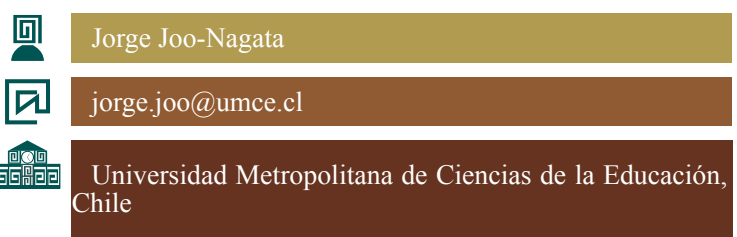




\section{INTRODUCCIÓN}

La Sociedad del Conocimiento, llamada así por algunos pensadores (Castells 2001; Evers 2003), ha modificado y continúa cambiando drásticamente varios aspectos del ser humano, a través de las denominadas Tecnologías de la Información y Comunicación (TIC) que conllevan en la actualidad un papel importante en la sociedad y en la cultura (Allen 2015), y por supuesto en la Educación, desde sus etapas iniciales hasta los niveles universitarios. Estos grupos poblacionales tienen un mayor acceso y uso de diversos dispositivos electrónicos como son computadores, teléfonos inteligentes y tabletas por mencionar algunos (International Telecomunications Union 2016), así como también el manejo de diversos recursos y herramientas disponibles en Internet (Vilhelmson, Thulin y Elldér 2017) y particularmente de la Web 2.0.

Por otro lado, las instituciones de educación superior, al contar con este nuevo tipo de estudiantes, debería aprovechar sus potencialidades y facilitar el desarrollo de nuevas competencias que no respondan solamente a aquellas previamente establecidas a través del currículo de tal o cual Carrera, sino que tengan la capacidad de adaptarse a los continuos cambios relacionados a su campo laboral o funciones inherentes con su práctica profesional, muchas de ellas todavía inexistentes (Humanante-Ramos 2016).

El desarrollo de competencias se plantea como un enfoque más cercano al mercado de trabajo (Arras, Torres y Garcia-Valcárcel 2011) y se puede decir que una persona competente, en determinada profesión, manifiesta a quien realiza bien lo que se espera de ella.

Al relacionar este concepto con el uso de las TIC, se puede decir que las Competencias Digitales del siglo XXI son el "conjunto de habilidades, competencias y conocimientos que los estudiantes debieran manejar para tener éxito en la sociedad contemporánea. Incluyen creatividad e innovación, pensamiento crítico, resolución de problemas, co- municación y colaboración, alfabetización informacional, alfabetización de medios y alfabetización de TIC". (Bilbao y Salinas 2010:217), es decir competente a la hora de realizar diseños en Web, manejar presentaciones, programas para elaborar gráficos, hojas de cálculo, bases de datos bibliográficas en línea, navegadores Web, programas de correo electrónico, aplicaciones para chat y procesadores de texto, marcadores sociales, sindicación de contenidos (RSS), redes sociales, entre otros.

Además, como afirma Cabero y Llorente: "En este nuevo entramado, se hace necesario que los sujetos estén capacitados para movilizar y utilizar las nuevas herramientas de comunicación que tienen a su disposición en la sociedad del conocimiento y la gran diversidad de signos que utilizan."(Cabero y Llorente 2008:11)

Para esto, se hace necesario el conocer las competencias TIC que poseen los estudiantes cuando llegan a una institución de nivel superior, con el fin de contar con un punto de partida dentro del cual se propongan los objetivos y los resultados de aprendizaje de las asignaturas relacionadas con el manejo de las TIC (Gisbert y Esteve 2016) o para el desarrollo de nuevas competencias transversales (Guitert, Guerrero, Ornellas, Romeu y Romero 2008).

En este trabajo de investigación se presentan los resultados de un estudio diagnóstico sobre las percepciones que tienen los estudiantes que ingresan a la Facultad de Ciencias de la Educación, de la Universidad Nacional de Chimborazo en Ecuador, sobre el manejo de sus competencias TIC.

\section{METODOLOGÍA}

La presente investigación se alinea dentro del paradigma de investigación cuantitativo, cuyo alcance es descriptivo debido a que se indaga la percepción que tienen los estudiantes universitarios sobre el manejo de sus competencias TIC. Además corresponde a un estudio de tipo no experimental debido a que "se realizan sin la manipulación deliberada 
de variables y en los que sólo se observan los fenómenos en su ambiente natural para después analizarlos" (Hernández, Fernández y Baptista 2010:149), además es transversal, porque el proceso de recolección de datos se realiza en un determinado momento del tiempo.

La población estuvo formada por los estudiantes de primer semestre de todas las $\mathrm{Ca}$ rreras de la Facultad de Ciencias de la Educación, Humanas y Tecnologías (FCEHyT), de la Universidad Nacional de Chimborazo (UNACH) en Ecuador y, de acuerdo al informe de la Unidad de Control Académico (UTECA) de esta universidad, los estudiantes matriculados en el primer semestre, en el ciclo académico abril 2017 - agosto 2017 son 188 estudiantes (UTECA 2017). Una distribución por Carreras se muestra en la Figura 1. Además, al ser un grupo pequeño, se trabajó con toda la población.

El instrumento utilizado fue el Cuestionario sobre competencias TIC de los estudiantes de primer semestre/año, el cual fue adaptado al contexto ecuatoriano a partir de un instrumento propuesto por la Universidad Metropolitana de Ciencias de la Educación de Chile, el mismo que fue elaborado tomando como referencia el trabajo de (Cabero, Llorente, y Marín 2010).

El mencionado instrumento contiene 75 ítems distribuidos en dos partes: una primera parte sobre posesión y uso de dispositivos y aplicaciones tecnológicas (39 ítems) y una segunda sobre sus autopercepciones en el uso de tecnología (36 ítems). La mayor parte de interrogantes corresponden a preguntas valoradas con una escala tipo Likert, por ejemplo, para un grupo de ítems el estudiante puede responder con valores de 1 a 5 , donde 1 completamente ineficaz, 3 moderadamente competente y 5 completamente competente, existiendo también la posibilidad de seleccionar la opción NC (No Conozco).

El valor mínimo aceptable para el coeficiente alfa de Cronbach es 0,70 ; por debajo de ese valor la consistencia interna de la escala utilizada es baja (Oviedo \& Campo Arias, 2005). Es decir, según este criterio el coeficiente se considera tolerable si supera el 0,70. En este trabajo el resultado fue de 0.958 que es considerado como excelente (George, Mallery 2003); por tanto el intrumento se consideró estadísticamente confiable y se procedió a la aplicación del mismo.

Este instrumento fue implementado utilizando los formularios de Google Docs y aplicado en línea durante la cuarta semana del mes de abril y la primera semana de mayo del 2017, al total de alumnos de las Carreras mencionadas anteriormente. Posteriormente los datos fueron procesados utilizando el software estadístico IBM SPSS 22.

Figura 1. Distribución por Carreras de los estudiantes de primer semestre FCEHyT-UNACH (N=188), Abril 2017 - Agosto 2017.

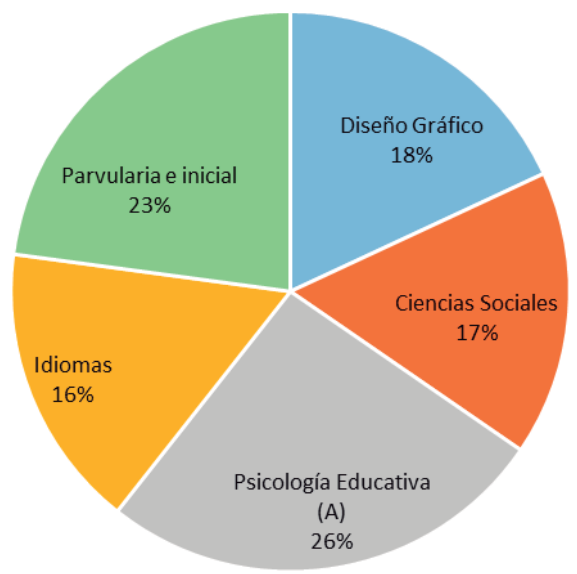

Fuente: UTECA (2017)

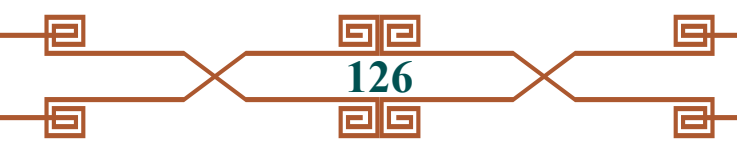




\section{RESULTADOS Y DISCUSIÓN}

De los 188 estudiantes que conformaron el universo del estudio, solamente respondieron al cuestionario 180, es decir el $96 \%$ de la población, cuyos principales resultados se describen a continuación.

Así, según se observa en la Tabla 1, el mayor porcentaje (62,8\% acumulado) de los estudiantes que se inician en el uso de un computador están entre los 6 y 10 años de edad en tanto que aproximadamente tres de cada 10 estudiantes (32,8\% acumulado) señalan que se han iniciado desde los 11 a 15 años de edad y solamente un 1,7\% (acumulado) dicen que han utilizado el computador antes de los 5 años de edad.

\section{Sobre la posesión y uso de dispositivos tecnológicos}

Para conocer acerca de la posesión y uso de los dispositivos tecnológicos, los cuales se han convertido en equipos de uso masivo por parte de los estudiantes, se han incluido aquellos que son de uso común, como: un computador de escritorio, un computador portátil, una impresora, un scanner, teléfo- nos celulares básicos, teléfonos celulares inteligentes (smartphones) y tabletas (tablets); pero también se incluyen otro tipo de dispositivos no tan comúnmente utilizados como una consola de videojuegos, los libros electrónicos o ebooks, y otros dispositivos que conozcan y sepan usar.

Los resultados indican que un $98,3 \%$ de los estudiantes consultados saben utilizar un computador de escritorio, un 95,6\% saben utilizar un computador portátil, igualmente una impresora con el 88,3\% y celular básico con el 95\%. Sin embargo, en cuanto al uso académico de estos dispositivos tecnológicos, como es el caso de los smartphones, se observa que el $44,4 \%$ no ha usado en actividades de estudio, o el $97,2 \%$ de usuarios de consolas de juego no lo ha hecho con fines académicos. Una mayor descripción de la posesión y uso de estos dispositivos se ilustra en la Tabla 2.

Conocido este primer escenario, sobre cuáles son los equipos que mayormente poseen y usan los estudiantes encuestados, se consultó sobre el tiempo de uso de los mismos, en número de horas. Los resultados muestran que el 44,4\% de los estudiantes usan el computador de escritorio menos de una hora

Tabla 1. Edad en la cual los estudiantes iniciaron el uso de un computador $(\mathrm{n}=180)$

\begin{tabular}{|c|c|c|}
\hline $\begin{array}{c}\text { Edad de inicialización } \\
\text { en el uso de las TIC }\end{array}$ & Frecuencia & Porcentaje \\
\hline 4 & 2 & $1,1 \%$ \\
\hline 5 & 1 & $0,6 \%$ \\
\hline 6 & 7 & $3,9 \%$ \\
\hline 7 & 17 & $9,4 \%$ \\
\hline 8 & 27 & $15,0 \%$ \\
\hline 9 & 21 & $11,7 \%$ \\
\hline 10 & 41 & $22,8 \%$ \\
\hline 11 & 9 & $5,0 \%$ \\
\hline 12 & 30 & $16,7 \%$ \\
\hline 13 & 9 & $5,0 \%$ \\
\hline 14 & 4 & $2,2 \%$ \\
\hline 15 & 7 & $3,9 \%$ \\
\hline 16 & 4 & $2,2 \%$ \\
\hline 20 & 1 & $0,6 \%$ \\
\hline Total & 180 & $100 \%$ \\
\hline
\end{tabular}

Fuente: Encuesta a estudiantes de primer semestre de las Carreras de la Facultad de Ciencias de la Educación-UNACH

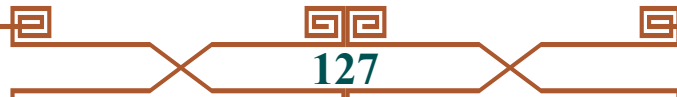


al día, en tanto que un $36,1 \%$ señalan que usan el computador portátil entre 3 a 4 horas al día.

En el caso de la impresora, el 85,6\% de los consultados, indican que su uso se reduce a menos de una hora al día, el scanner igualmente menos de una hora diaria (95.6\%). El smartphone, es usado menos de una hora al día por el un $33,9 \%$ de los encuestados, sin embargo, el $47 \%$ de estudiantes usan entre tres y ocho horas diarias.

Finalmente, dispositivos como la tablet, la consola de videojuegos, y el ebook; un promedio del $75 \%$ de los encuestados usan menos de una hora al día. Una descripción detallada de esta información se presenta en la Tabla 3.

\section{Sobre el uso de aplicaciones de Internet y redes sociales}

Revisados los datos sobre el uso de dispositivos tecnológicos, en esta parte, se presenta información sobre cuáles son las aplicaciones informáticas disponibles en la web ma- yormente usadas por los estudiantes. Para esto, se incluye varias aplicaciones como: el correo electrónico, el chat, Facebook, Twitter, Instagram, Snapchat, Tumblr, Flickr, entre otras.

Los resultados indican que Snapchat si bien conocen el $68,9 \%$, pero un $57.2 \%$ no saben utilizarla, lo mismo sucede con Twitter que conocen el $80,6 \%$ pero un $59,4 \%$ no saben utilizarlo. Otras aplicaciones, como Tumblr, desconocen el $77,8 \%$ de los encuestados y no saben utilizarlo el $91,7 \%$, al igual que Flickr en un $91,7 \%$ desconocen y un $98,9 \%$ no utilizan. Un resumen de esta información se presenta en la Tabla 4.

\section{Sobre el uso de aplicaciones para tra- bajo colaborativo y almacenamiento en la nube}

En cuanto al uso de aplicaciones informáticas para el trabajo colaborativo, así como aquellas que permiten almacenar información en la nube (cloud computing), se incluyeron en la consulta algunas herramientas como:

Tabla 2. Posesión y Uso de dispositivos tecnológicos en actividades personales y académicas $(\mathrm{n}=180)$

\begin{tabular}{|l|c|c|c|c|c|c}
\hline \multirow{2}{*}{ Equipo } & \multicolumn{2}{c|}{ Tiene } & \multicolumn{2}{c|}{ iSabes Usar? } & \multicolumn{2}{c}{$\begin{array}{c}\text { iHas usado esta } \\
\text { aplicación para las } \\
\text { actividades de estudio? }\end{array}$} \\
\cline { 2 - 7 } & SI & NO & SI & NO & SI & NO \\
\hline $\begin{array}{l}\text { Computador } \\
\text { escritorio }\end{array}$ & $53,3 \%$ & $46,7 \%$ & $98,3 \%$ & $1,7 \%$ & $88,9 \%$ & $11,1 \%$ \\
\hline $\begin{array}{l}\text { Computador } \\
\text { Portátil }\end{array}$ & $70,6 \%$ & $29,4 \%$ & $95,6 \%$ & $4,4 \%$ & $89,4 \%$ & $10,6 \%$ \\
\hline Impresora & $53,9 \%$ & $46,1 \%$ & $88,3 \%$ & $11,7 \%$ & $94,4 \%$ & $5,6 \%$ \\
\hline Scanner & $42,8 \%$ & $57,2 \%$ & $63,9 \%$ & $36,1 \%$ & $87,8 \%$ & $12,2 \%$ \\
\hline $\begin{array}{l}\text { Celular } \\
\text { básico }\end{array}$ & $58,9 \%$ & $41,1 \%$ & $95,0 \%$ & $5,0 \%$ & $40,6 \%$ & $59,4 \%$ \\
\hline Smartphone & $60,6 \%$ & $39,4 \%$ & $78,3 \%$ & $21,7 \%$ & $55,6 \%$ & $44,4 \%$ \\
\hline $\begin{array}{l}\text { Consola de } \\
\text { videojuegos }\end{array}$ & $22,2 \%$ & $77,8 \%$ & $51,1 \%$ & $48,9 \%$ & $2,8 \%$ & $97,2 \%$ \\
\hline Tablet & $28,9 \%$ & $71,1 \%$ & $82,8 \%$ & $17,2 \%$ & $42,8 \%$ & $57,2 \%$ \\
\hline Ebook & $13,9 \%$ & $86,1 \%$ & $29,4 \%$ & $70,6 \%$ & $42,2 \%$ & $57,8 \%$ \\
\hline Otros & $35,6 \%$ & $64,4 \%$ & $53,9 \%$ & $46,1 \%$ & $41,1 \%$ & $58,9 \%$ \\
\hline Funyyyyyyy
\end{tabular}

Fuente: Encuesta a estudiantes de primer semestre de las Carreras de la Facultad de Ciencias de la Educación-UNACH 
Tabla 3. Tiempo de uso de dispositivos tecnológicos $(\mathrm{n}=180)$

\begin{tabular}{|l|c|c|c|c|c|c|}
\hline \multicolumn{1}{|c|}{ Equipo } & $1-2$ horas & $\begin{array}{c}3-4 \\
\text { horas }\end{array}$ & $\begin{array}{c}5-6 \\
\text { horas }\end{array}$ & $\begin{array}{c}7-8 \\
\text { horas }\end{array}$ & $\begin{array}{c}>8 \\
\text { horas }\end{array}$ & $\begin{array}{c}<1 \\
\text { hora }\end{array}$ \\
\hline $\begin{array}{l}\text { Computador de } \\
\text { escritorio }\end{array}$ & $32,2 \%$ & $17,2 \%$ & $3,3 \%$ & $1,7 \%$ & $1,1 \%$ & $44,4 \%$ \\
\hline Computador Portátil & $26,1 \%$ & $36,1 \%$ & $11,7 \%$ & $3,9 \%$ & $1,7 \%$ & $20,6 \%$ \\
\hline Impresora & $10,6 \%$ & $1,7 \%$ & $1,7 \%$ & $0,6 \%$ & $0,0 \%$ & $85,6 \%$ \\
\hline Scanner & $2,8 \%$ & $1,1 \%$ & $0,6 \%$ & $0,0 \%$ & $0,0 \%$ & $95,6 \%$ \\
\hline Celular básico & $8,9 \%$ & $13,9 \%$ & $4,4 \%$ & $3,3 \%$ & $4,4 \%$ & $65,0 \%$ \\
\hline Smartphone & $6,7 \%$ & $15,0 \%$ & $17,2 \%$ & $9,4 \%$ & $17,8 \%$ & $33,9 \%$ \\
\hline $\begin{array}{l}\text { Consola de } \\
\text { videojuegos }\end{array}$ & $7,2 \%$ & $5,0 \%$ & $1,7 \%$ & $2,2 \%$ & $0,6 \%$ & $83,3 \%$ \\
\hline Tablet & $11,7 \%$ & $9,4 \%$ & $5,0 \%$ & $1,1 \%$ & $0,0 \%$ & $72,8 \%$ \\
\hline Ebook & $11,1 \%$ & $6,7 \%$ & $1,7 \%$ & $0,0 \%$ & $1,7 \%$ & $78,9 \%$ \\
\hline Otros & $10,0 \%$ & $6,7 \%$ & $2,8 \%$ & $2,8 \%$ & $2,2 \%$ & $75,6 \%$ \\
\hline
\end{tabular}

Fuente: Encuesta a estudiantes de primer semestre de las Carreras de la Facultad de Ciencias de la Educación-UNACH

Dropbox, Google Drive, ICloud, OneDrive y Mega, dejando también la posibilidad de que el encuestado mencione otras similares que conozca y use. Así, a nivel general se observa que, ICloud, desconocen 7 de cada 10 encuestados, al igual que la herramienta Mega, que es desconocida por cerca del 64\% de los estudiantes.
En cambio, la herramienta que más conocen los estudiantes es Google Drive por más del $90 \%$ de los encuestados, seguida de DropBox con el $65.6 \%$. Esta tendencia se mantiene cuando se consulta sobre si saben utilizar o no estas herramientas, sin embargo, un dato que llama la atención es el caso de que la herramienta DropBox, que es conocida

Tabla 4. Uso de aplicaciones de Internet y Redes sociales $(\mathrm{n}=180)$

\begin{tabular}{|l|c|c|c|c|}
\hline \multirow{2}{*}{ Herramienta } & \multicolumn{2}{c|}{ Conoce } & \multicolumn{2}{c|}{ Utiliza } \\
\cline { 2 - 5 } & SI & NO & SI & NO \\
\hline Correo Electrónico & $100,0 \%$ & $0,0 \%$ & $99,4 \%$ & $0,6 \%$ \\
\hline Chat & $98,9 \%$ & $1,1 \%$ & $95,0 \%$ & $5,0 \%$ \\
\hline Facebook & $100,0 \%$ & $0,0 \%$ & $96,7 \%$ & $3,3 \%$ \\
\hline Twitter & $80,6 \%$ & $19,4 \%$ & $40,6 \%$ & $59,4 \%$ \\
\hline Instagram & $86,7 \%$ & $13,3 \%$ & $62,8 \%$ & $37,2 \%$ \\
\hline Snapchat & $68,9 \%$ & $31,1 \%$ & $42,8 \%$ & $57,2 \%$ \\
\hline Tumblr & $22,2 \%$ & $77,8 \%$ & $8,3 \%$ & $91,7 \%$ \\
\hline Flickr & $8,3 \%$ & $91,7 \%$ & $1,1 \%$ & $98,9 \%$ \\
\hline Otras aplicaciones & $50,0 \%$ & $50,0 \%$ & $42,8 \%$ & $57,2 \%$ \\
\hline
\end{tabular}

Fuente: Encuesta a estudiantes de primer semestre de las Carreras de la Facultad de Ciencias de la Educación-UNACH 
por una buena parte de los encuestados, pero sólo saben utilizar 1 de cada 2 estudiantes. En cuanto al uso de estas aplicaciones para las actividades de estudio, se observa que la herramienta mayormente usada es Google Drive por 8 de cada 10 consultados. Un resumen de estos porcentajes se presenta en la Tabla 5.

\section{Sobre el uso de aplicaciones para tomar notas}

En cuanto al uso de aplicaciones para tomar notas, se consideraron algunas herramientas informáticas como alternativas a los tradicionales procesadores de texto como: EverNote, OneDrive, Notas IPhone, Notas Android, dejando la posibilidad de que el encuestado mencione otras similares que conozca y use. Así, los procesadores de texto (Microsoft Word, Writer, entre otros), son los programas informáticos que mayormente conocen y usan para tomar notas con fines académicos por más del $92 \%$ de los encuestados.

Sin embargo, aproximadamente el $78 \%$ de los encuestados indican que también conocen y usan las Notas de sus teléfonos Android, donde 7 de cada 10 estudiantes lo hace con fines de estudio, siendo menos usadas para esta función las herramientas de notas de los teléfonos IPhone. También, dentro de esta categoría, la herramienta más desconocida para los encuestados es Evernote, la cual además es usada con fines académicos por apenas el $6,10 \%$ de los estudiantes consultados. Un resumen de los porcentajes de estos resultados se incluye en la Tabla 6 .

\section{Sobre la generación de contenidos di- gitales}

La difusión de contenidos digitales a través de herramientas de la Web 2.0, fue otro de los aspectos consultados, así, se observa que herramientas como YouTube y Facebook son conocidas por prácticamente la totalidad de encuestados (100\%), seguidas de Instagram y Twitter conocidas por más del $80 \%$ de los estudiantes consultados. En cambio, la herramienta Pinterest es desconocida para 7 de cada 10 estudiantes que participaron de la encuesta, esto se puede observar en la Figura 2.

\section{Sobre la autopercepción en el uso de tecnología}

Un elemento adicional y motivo de análisis fue el conocer la autopercepción que tiene el estudiante con respecto al uso y conocimiento de la tecnología, en este sentido se indagaron sobre varios aspectos, como se pude observar en los resultados que se presentan en la Tabla 7.

Así, partiendo del análisis de lo presentado en la Tabla 7 y del cálculo de la moda aplicada a cada variable se pudo determinar que los

Tabla 5. Uso de aplicaciones informáticas para el trabajo colaborativo y para almacenamiento en la nube $(\mathrm{n}=180)$

\begin{tabular}{|l|r|r|r|r|r|r|}
\hline \multirow{2}{*}{ Herramienta } & \multicolumn{2}{|c|}{ ¿Conoces? } & \multicolumn{2}{c|}{ ¿Sabes Usar? } & \multicolumn{2}{c|}{$\begin{array}{c}\text { ¿Has usado esta } \\
\text { aplicación para las } \\
\text { actividades de estudio? }\end{array}$} \\
\cline { 2 - 7 } & SI & \multicolumn{1}{|c|}{ NO } & SI & NO & SI & NO \\
\hline DropBox & $65,6 \%$ & $34,4 \%$ & $50,6 \%$ & $49,4 \%$ & $49,4 \%$ & $50,6 \%$ \\
\hline Google Drive & $92,8 \%$ & $7,2 \%$ & $82,8 \%$ & $17,2 \%$ & $82,8 \%$ & $17,2 \%$ \\
\hline ICloud & $30,0 \%$ & $70,0 \%$ & $21,7 \%$ & $78,3 \%$ & $10,6 \%$ & $89,4 \%$ \\
\hline OneDrive & $58,9 \%$ & $41,1 \%$ & $37,8 \%$ & $62,2 \%$ & $35,6 \%$ & $64,4 \%$ \\
\hline Mega & $36,1 \%$ & $63,9 \%$ & $27,8 \%$ & $72,2 \%$ & $18,3 \%$ & $81,7 \%$ \\
\hline Otras & $41,1 \%$ & $58,9 \%$ & $33,9 \%$ & $66,1 \%$ & $33,3 \%$ & $66,7 \%$ \\
\hline
\end{tabular}

Fuente: Encuesta a estudiantes de primer semestre de las Carreras de la Facultad de Ciencias de la Educación-UNACH 
Tabla 6. Uso de aplicaciones para tomar notas $(n=180)$

\begin{tabular}{|l|r|r|r|r|r|r|}
\hline \multirow{2}{*}{ Herramienta } & \multicolumn{2}{|c|}{ ¿Conoces? } & \multicolumn{2}{c|}{ ¿Sabes Utilizar? } & \multicolumn{2}{c|}{$\begin{array}{c}\text { ¿Has usado esta } \\
\text { aplicación para las } \\
\text { actividades de } \\
\text { estudio? }\end{array}$} \\
\cline { 2 - 8 } & \multicolumn{1}{|c|}{ SI } & \multicolumn{1}{|c|}{ NO } & \multicolumn{1}{c|}{ SI } & \multicolumn{1}{c|}{ NO } & \multicolumn{1}{c|}{ SI } & \multicolumn{1}{c|}{ NO } \\
\hline EverNote & $8,9 \%$ & $91,1 \%$ & $7,2 \%$ & $92,8 \%$ & $6,1 \%$ & $93,9 \%$ \\
\hline OneDrive & $57,2 \%$ & $42,8 \%$ & $48,3 \%$ & $51,7 \%$ & $47,2 \%$ & $52,8 \%$ \\
\hline $\begin{array}{l}\text { Procesadores de } \\
\text { Texto }\end{array}$ & $92,8 \%$ & $7,2 \%$ & $92,8 \%$ & $7,2 \%$ & $92,2 \%$ & $7,8 \%$ \\
\hline Notas IPhone & $48,3 \%$ & $51,7 \%$ & $46,7 \%$ & $53,3 \%$ & $38,3 \%$ & $61,7 \%$ \\
\hline Notas Android & $78,3 \%$ & $21,7 \%$ & $77,8 \%$ & $22,2 \%$ & $72,8 \%$ & $27,2 \%$ \\
\hline Otras & $52,8 \%$ & $47,2 \%$ & $50,0 \%$ & $50,0 \%$ & $46,1 \%$ & $53,9 \%$ \\
\hline
\end{tabular}

Fuente: Encuesta a estudiantes de primer semestre de las Carreras de la Facultad de Ciencias de la Educación-UNACH

estudiantes son completamente competentes en el conocimiento y uso de un $17 \%$ (siete de 36) de las competencias investigadas correspondientes a: la edición básica de documentos de texto, realizar descargas de archivos desde el internet, navegar fácilmente en internet, comunicarse fácilmente con otras personas por medio virtuales (chat, email), utilizar diferentes browsers para navegar en internet y enviar archivos de un computador a otro por internet.

Por otro lado, afirman ser competentes en un $6 \%$ (dos de 36) de las competencias descritas como: conocer el funcionamiento básico del computador y crear presentaciones multimedia. Son moderadamente competentes en el
$72 \%$ (26 de 36) de actividades tales como: diseñar y/o modificar el contenido de una hoja cálculo; organizar información recogida del Internet, utilizar plataformas virtuales educativas; aportar crítica y positivamente en la producción multimedia; explicar las ventajas y limitaciones del uso del computador y la red para gestionar información; conocer los recursos electrónicos disponibles en la biblioteca electrónica de la UNACH; formar parte de comunidades virtuales; crear imágenes y gráficos con ayuda de PC; por mencionar algunas incluidas en este grupo, un listado completo de estas competencias con valor de moda es igual a 3 , se presenta en la Tabla 7.

Figura 2. Sobre si conocen o no herramientas para la generación de contenidos digitales $(n=180)$.

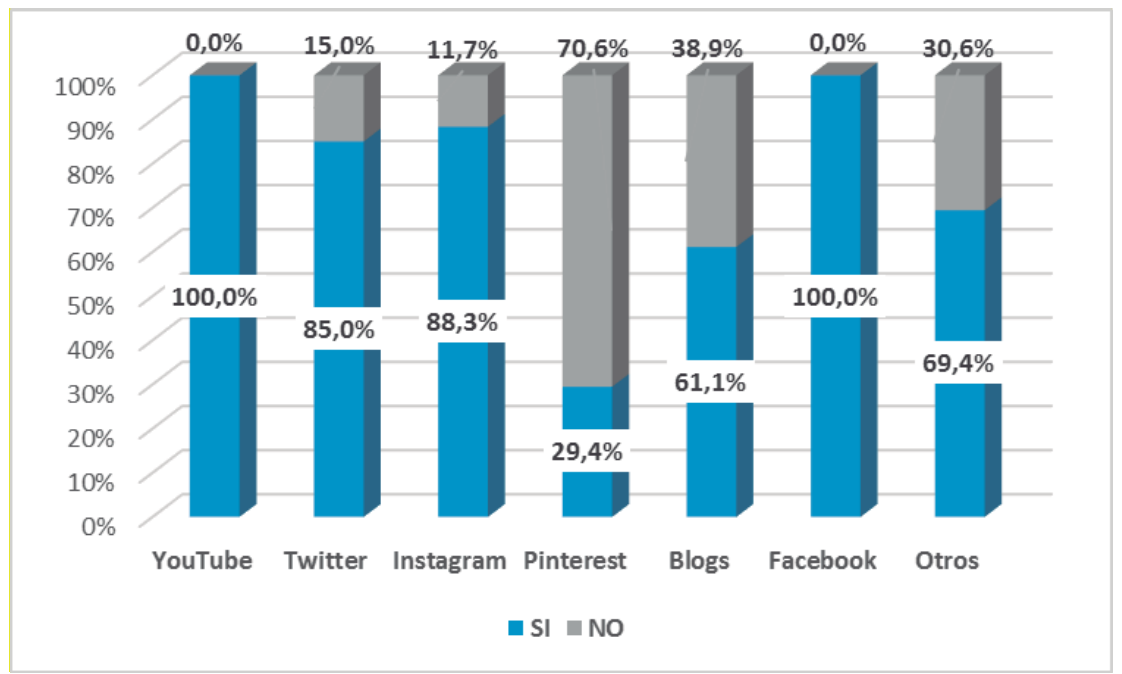


Finalmente, los estudiantes encuestados desconocen o son completamente ineficaces en el diseño y gestión base de datos, y en el desarrollo de páginas web, las cuales corresponden a un $6 \%$ (dos de 36$)$ de las competencias investigadas.
De manera generalizada, los estudiantes poseen una percepción general del uso de dispositivos tecnológicos propios de su nivel de competencia digital, es decir, su desempeño en el uso de estos dispositivos va siendo parte de su cotidianidad, como el uso de aquellos

Tabla 7. Autopercepción de los estudiantes sobre el uso de la tecnología $(n=180)$

\begin{tabular}{|c|c|c|c|c|c|}
\hline \multirow[b]{2}{*}{$\begin{array}{l}\text { VARIABLE } \\
\text { (competencia) }\end{array}$} & \multicolumn{5}{|c|}{ Medidas aplicadas } \\
\hline & $\begin{array}{l}\text { Media } \\
\text { Aritmética }\end{array}$ & $\begin{array}{l}\text { Desviación } \\
\text { Estándar }\end{array}$ & $\begin{array}{c}\text { Mod } \\
\text { a }\end{array}$ & $\begin{array}{c}\text { Datos } \\
\text { que } \\
\text { están } \\
\text { sobre la } \\
\text { media }\end{array}$ & $\begin{array}{l}\text { Porcentaj } \\
\text { e de datos } \\
\text { sobre la } \\
\text { media }\end{array}$ \\
\hline Edición básica de documentos de texto & 3,99 & 1,1699 & 5 & 122 & $68 \%$ \\
\hline $\begin{array}{l}\text { Realiza descargas de archivos desde el } \\
\text { internet }\end{array}$ & 3,95 & 1,1878 & 5 & 122 & $68 \%$ \\
\hline Navega fácilmente en internet & 3,67 & 1,1763 & 5 & 104 & $58 \%$ \\
\hline $\begin{array}{l}\text { Se comunica fácilmente con otras } \\
\text { personas por email, chat, etc. }\end{array}$ & 4,08 & 1,2393 & 5 & 98 & $54 \%$ \\
\hline $\begin{array}{l}\text { Utiliza diferentes browsers para navegar } \\
\text { en internet }\end{array}$ & 4,04 & 1,1548 & 5 & 87 & $48 \%$ \\
\hline $\begin{array}{l}\text { Envía archivos de un computador a otro } \\
\text { por internet }\end{array}$ & 3,38 & 1,3507 & 5 & 83 & $46 \%$ \\
\hline $\begin{array}{l}\text { Conoce el funcionamiento básico del } \\
\text { computador }\end{array}$ & 3,84 & 0,8334 & 4 & 111 & $62 \%$ \\
\hline Crea presentaciones multimedia & 3,18 & 1,2573 & 4 & 80 & $44 \%$ \\
\hline $\begin{array}{l}\text { Diseña y/o modifica el contenido de una } \\
\text { hoja cálculo }\end{array}$ & 2,94 & 1,1614 & 3 & 120 & $67 \%$ \\
\hline $\begin{array}{l}\text { Organiza información recogida del } \\
\text { internet }\end{array}$ & 2,97 & 1,2747 & 3 & 115 & $64 \%$ \\
\hline Utiliza plataformas virtuales educativas & 2,93 & 1,3330 & 3 & 115 & $64 \%$ \\
\hline $\begin{array}{l}\text { Conoce y respeta los derechos autor en } \\
\text { cuanto al uso de software }\end{array}$ & 2,90 & 1,2511 & 3 & 116 & $64 \%$ \\
\hline $\begin{array}{l}\text { Aporta crítica y positivamente en la } \\
\text { producción multimedia }\end{array}$ & 2,74 & 1,0471 & 3 & 114 & $63 \%$ \\
\hline $\begin{array}{l}\text { Explica las ventajas y limitaciones del } \\
\text { uso del computador y la red para } \\
\text { gestionar información }\end{array}$ & 2,81 & 1,1041 & 3 & 112 & $62 \%$ \\
\hline $\begin{array}{l}\text { Conoce los recursos electrónicos } \\
\text { disponibles en la biblioteca electrónica } \\
\text { de la UNACH }\end{array}$ & 2,84 & 1,1810 & 3 & 108 & $60 \%$ \\
\hline Forma parte de comunidades virtuales & 2,75 & 1,3614 & 3 & 102 & $57 \%$ \\
\hline Crea imágenes y gráficos & 2,68 & 1,2175 & 3 & 100 & $56 \%$ \\
\hline Modifica imágenes & 2,74 & 1,3344 & 3 & 99 & $55 \%$ \\
\hline $\begin{array}{l}\text { Diseña, publica y/o mantiene páginas } \\
\text { web personales (blogs, muros en redes } \\
\text { sociales) }\end{array}$ & 2,70 & 1,2636 & 3 & 98 & $54 \%$ \\
\hline $\begin{array}{l}\text { Conoce y navega en la webpage } \\
\text { institucional }\end{array}$ & 3,54 & 1,1498 & 3 & 96 & $53 \%$ \\
\hline Conecta equipos de audio y video & 3,52 & 1,1746 & 3 & 91 & $51 \%$ \\
\hline 04. Convierte el formato de archivos & 3,52 & 1,1408 & 3 & 91 & $51 \%$ \\
\hline $\begin{array}{l}\text { Utiliza las TICS para explorar e } \\
\text { investigar }\end{array}$ & 3,44 & 1,1923 & 3 & 88 & $49 \%$ \\
\hline
\end{tabular}




\begin{tabular}{|l|r|r|r|r|r|}
\hline $\begin{array}{l}\text { Maneja programas para compartir } \\
\text { virtualmente información con amigos y } \\
\text { familiares }\end{array}$ & 3,34 & 1,2198 & 3 & 83 & $46 \%$ \\
\hline $\begin{array}{l}\text { Organiza, analiza y sintetiza la } \\
\text { información mediante tablas, gráficos o } \\
\text { esquemas mentales con ayuda el PC }\end{array}$ & 3,23 & 1,1619 & 3 & 77 & $43 \%$ \\
\hline $\begin{array}{l}\text { Conoce las consecuencias del mal uso de } \\
\text { las TIC y aplica normas éticas para } \\
\text { evitarlo }\end{array}$ & & & & & \\
\hline $\begin{array}{l}\text { Utiliza herramientas online para trabajos } \\
\text { grupales }\end{array}$ & 3,20 & 1,3134 & 3 & 77 & $43 \%$ \\
\hline $\begin{array}{l}\text { Encuentra en la red datos de autores y } \\
\text { los cita en un trabajo correctamente }\end{array}$ & 3,14 & 1,2468 & 3 & 76 & $42 \%$ \\
\hline Instala y desinstala programas & 3,16 & 1,1918 & 3 & 75 & $42 \%$ \\
\hline $\begin{array}{l}\text { Maneja programas para compartir } \\
\text { virtualmente información con docentes } \\
\text { compañeros }\end{array}$ & 3,18 & 1,2617 & 3 & 74 & $41 \%$ \\
\hline $\begin{array}{l}\text { Utiliza herramientas para socializar } \\
\text { información personal y/o profesional }\end{array}$ & 3,15 & 1,1359 & 3 & 71 & $39 \%$ \\
\hline $\begin{array}{l}\text { Utiliza estrategias para navegar en } \\
\text { internet y publicar información personal } \\
\text { de manera segura }\end{array}$ & 3,14 & 1,2271 & 3 & 68 & $38 \%$ \\
\hline $\begin{array}{l}\text { Aplica criterios para seleccionar de } \\
\text { manera autónoma herramientas } \\
\text { educativas }\end{array}$ & & & & & $41 \%$ \\
\hline $\begin{array}{l}\text { Evalúa correctamente la relevancia y } \\
\text { fiabilidad de la información publicada en } \\
\text { internet. }\end{array}$ & 3,07 & 1,1629 & 3 & 62 & $34 \%$ \\
\hline Diseña y gestiona base de datos & 2,29 & 1,2213 & 1 & 72 & $40 \%$ \\
\hline Desarrollar páginas y sitios web & 2,18 & 1,2289 & 1 & 66 & $37 \%$ \\
\hline
\end{tabular}

NOTA: Escala utilizada tipo Likert, con valores de 1 a 5, donde 1 completamente ineficaz, 3 moderadamente competente y 5 completamente competente

artefactos de hogar que no requieren mayor conocimiento y que se usan mecánicamente. Además, se observa que a nivel académico existe aún la necesidad de sacar provecho al uso de estos dispositivos.

Si se analizan los resultados de ¿cuáles son los dispositivos? que van marcando tendencias de uso en la actual sociedad, existe una brecha entre lo que representa conocer y saber utilizar académicamente en el aula, como es el caso de una tablet.

Mientras que, el tiempo de uso de estos dispositivos tecnológicos, según señalan los estudiantes consultados, en su mayoría, es menos de una hora; queda evidenciado también el hecho de que los dispositivos móviles como los smartphones son aquellos que mayor tiempo los usan, pero no con fines académicos, y es ahí donde se debería orientar estas competencias de uso hacia competen- cias didácticas dentro del aprendizaje móvil o m-learning.

De la misma manera, el importante uso no académico de aplicaciones de internet y de redes sociales, abren varias oportunidades de utilización de estas herramientas en el campo educativo y que deberían ser consideradas como nuevas estrategias de aprendizaje. Los resultados del trabajo de investigación realizado, en cuanto a la forma como los estudiantes gestionan su trabajo colaborativo por medio del uso de herramientas informáticas y de alojamiento en la nube, indica que la herramienta Google Drive constituye el entorno digital, que los estudiantes mayormente conocen, saben usar y donde han realizado actividades de estudio.

Algo similar, se observa en otro trabajo realizado por (Álvarez, Sánchez 2014) donde se presenta la valoración positiva del uso de 
esta herramienta por parte de un grupo de estudiantes del Instituto Príncipe de Asturias en Lorca (España), en el desarrollo del aprendizaje cooperativo, su utilidad y la influencia en la motivación se sus aprendizajes.

El criterio del grupo de estudiantes consultado, también concuerda con otras experiencias sobre el uso de Google Drive en actividades colaborativas en la Universidad Internacional de la Rioja, España (Castellanos, Martínez 2013), en el cual los estudiantes participantes destacan la facilidad del manejo de la herramienta, la sencillez en la formación de grupos de trabajo online y la utilidad pedagógica para las tareas educativas.

De igual forma, se evidencia un avance en el desarrollo de herramientas útiles en la facilitación de trabajo colaborativo si las comparamos con experiencias de hace 10 años atrás, donde, para soportar la comunicación online en el trabajo colaborativo se utilizaba herramientas como: páginas web, grupos de discusión y correo electrónico (Cenich, Santos 2005).

Los procesos de documentación de las clases presenciales o virtuales por parte de los estudiantes, así como también las notas que se toman cuando asisten a algún evento académico como conferencias, congresos, simposios, entre otros, siguen siendo realizados por medio de programas procesadores de texto. Sin embargo, resulta sumamente importante analizar el potencial que representan las aplicaciones de notas de los smartphones, usados por una gran parte de estudiantes. En este punto, los resultados también se respaldan de las tendencias a nivel mundial en cuanto al uso masivo de tecnologías Android en los smartphone en comparación con los teléfono IPhone (Ascheri, Testa, Pizarro, Camiletti y Diaz 2014).

El conocer las oportunidades que ofrecen las herramientas Web 2.0 como medios para la generación de contenidos digitales (Torre 2006) es una realidad en el contexto educativo analizado, siendo las herramientas como Facebook, YouTube, Instagram las mayormente ubicadas para estos fines, seguidas muy de cerca de la herramienta Twitter, sin embargo, algo que sí llama la atención, es el hecho de que recursos como los Blogs no sean conocidos por la totalidad de los encuestados, desaprovechando las muchas potencialidades que ofrecen este tipo de herramientas no solo para la socialización de contenidos, sino como medios para la generación de espacios de discusión y construcción de conocimiento colaborativo (Cabero, López y Ballesteros 2009; Lara 2005).

Como se puede notar, los estudiantes realizan con mucha facilidad y experticia actividades tales como: editar documentos de texto, navegar y descargar archivos del internet, comunicarse virtualmente con otras personas, enviar archivos de un computador a otro, emplear diferentes tipos de browser, habilidades que han sido generadas en la experiencia cotidiana y en las aulas de sus colegios. Es normal encontrar que, actividades como la gestión de base de datos y desarrollo de páginas web sean desconocidas para los estudiantes, puesto que, dentro del contexto estudiado, solamente en los colegios que ofertan bachilleratos con especialidad en Informática, suelen abordarse este tipo de temáticas.

\section{CONCLUSIONES}

Aunque por la misma naturaleza de esta investigación no se podrían establecer generalizaciones, sí existe la posibilidad de exponer algunas conclusiones importantes para el colectivo académico estudiado.

Así, se observó que los estudiantes, a partir de los 4 años de edad ya han empezado a utilizar diversos dispositivos electrónicos, pero es, entre los 7 y 10 años de edad, donde más de la mitad de estudiantes se inicializa en el uso de las TIC.

Sin embargo, en la actualidad el uso de los dispositivos electrónicos es diverso, ya que mayormente son las computadoras portátiles y los teléfonos celulares inteligentes o smartphones.

Además, se evidencia en el contexto educativo consultado, el predominio del sistema operativo móvil Android sobre IOS de Apple; aunque, el desarrollo y uso de aplicaciones 
educativas no debería excluir a ninguno de los dos grupos de usuarios.

Estos recursos tecnológicos se han convertido en elementos de uso diario, al punto de que, seis de cada 10 estudiantes afirman utilizar sus computadoras portátiles, entre una y cuatro horas al día y si comparamos con el tiempo que asisten a sus clases presenciales, se puede entender el potencial que representa, en el contexto educativo estudiado, el poder apoyar la docencia presencial con actividades de aprendizaje electrónico o virtual. Por otro lado, en cuanto al uso de aplicaciones de las TIC, la mayoría de estudiantes universitarios lo hacen en actividades no necesariamente académicas, siendo en este sentido, oportuno fomentar el uso de herramientas informáticas como Google Drive para apoyar sus procesos educativos, como es el caso del desarrollo de espacios de aprendizaje colaborativo, o también para la gestión de su información online a través del uso de otros recursos como DropBox y One Drive.

En general, se puede apreciar que los estudiantes afirman ser moderadamente competentes en el uso de la tecnología, lo que les permite empezar sus Carreras universitarias con un nivel básico en el manejo de recursos y aplicaciones informáticas para la edición de documentos, navegar y descargar archivos del internet, y para comunicarse mediante medios virtuales.

Estos resultados dejan abierta la posibilidad de plantear actividades que potencialicen el uso eficiente de las tecnologías no solamente con el trabajo académico que deben cumplir los estudiantes sino también con el fin de mejorar sus competencias digitales tan necesarias para su ejercicio profesional en esta sociedad del conocimiento.

\section{REFERENCIAS BIBLIOGRÁFICAS}

Allen, L. E. (2015). The influence of information and communication technologies on societies and their cultures: A historical perspective. En Handbook of Research on Sustainable Development and Economics (pp. 360-385). https://doi. org/10.4018/978-1-4666-8433-1.ch016

Álvarez Ferrón, M., \& Sánchez Cañizares, L. (2014). Conocimiento, valoración y utilización, por parte del alumnado, de "Google Drive» como herramienta de trabajo cooperativo. Recuperado de https://gredos.usal.es/jspui/handle/10366/129553

Arras, A., Torres, C., \& Garcia-Valcárcel, A. (2011). Competencias en Tecnologías de Información y. Revista Latina de Comunicación Social, 66, 1-26.

Ascheri, M. E., Testa, O., Pizarro, R. A., Camiletti, P., \& Diaz, L. (2014). Desarrollo de aplicaciones para dispositivos móviles con sistema operativo Android para la enseñanza aprendizaje de temas de matemáticas en el nivel medio (pp. 1093-1098). Presentado en XVI Workshop de Investigadores en Ciencias de la Computación, Tierra de Fuego, Argentina. Recuperado a partir de http://hdl.handle. net/10915/43878

Bilbao, A., \& Salinas, A. (Eds.). (2010). El Libro Abierto de la Informática Educativa. Santiago de Chile, Chile: Enlaces.

Cabero, J., Llorente, M. del C., \& Marín, V. (2010). Hacia el diseño de un instrumento de diagnóstico de competencias tecnológicas del profesorado" universitario, Revista Iberoamericana de Educación, 52 (7), 1-12.

Cabero-Almenara, J., \& Llorente Cejudo, C. (2008). La alfabetización digital de los alumnos. Competencias digitales para el siglo XXI. Revista Portuguesa de Pedagogia, 42 (2), 7-28.

Cabero-Almenara, J., López Meneses, E., \& Ballesteros Regaña, C. (2009). Experiencias universitarias innovadoras con blogs para la mejora de... Revista Universidad y Sociedad del Conocimiento, 6 (2). Recuperado de http://www.redalyc.org/ html/780/78012947003/

Castellanos Sánchez, A., \& Martínez De la Muela, A. (2013). Trabajo en equipo con Google Drive en la universidad online. Innovación educativa, 13 (63), 75-94

Castells, M. (2001). The Internet Galaxy: Reflections on the Internet, Business, and Society. Oxford: Oxford University Press.

Cenich, G., \& Santos, G. (2005). Propuesta de aprendizaje basado en proyecto y trabajo colaborativo: experiencia de un curso en línea. Revista electrónica de investigación educativa, 7 (2), 1-18.

Evers, H.-D. (2003). Transition towards a Knowledge Society: Malaysia and Indonesia in Comparative 
Perspective. Comparative Sociology, 2 (2), 355-373. https://doi.org/10.1163/156913303100418816

George, D., \& Mallery, P. (2003). SPSS for Windows Step by Step: A Simple Guide and Reference (4ta ed.). Boston: Allyn \& Bacon. Recuperado de https:// books.google.es/books?id=AghHAAAAMAAJ\&source=gbs_book_other_versions

Gisbert, M., \& Esteve, F. (2016). Digital Leaners: la competencia digital de los estudiantes universitarios. La Cuestión Universitaria, 0 (7), 48-59.

Guitert, M., Guerrero, A. E., Ornellas, A., Romeu, T., \& Romero, M. (2008). Implementación de la competencia transversal "Uso y aplicación de las TIC en el ámbito académico y profesional» en el contexto universitario de la UOC. Recuperado de http://dehesa. unex.es/xmlui/handle/10662/1309

Hernández, R., Fernández, C., \& Baptista, P. (2010). Metodología de la investigación. México DF, México: McGrawHill.

Humanante Ramos, P. R. (2016). Entornos Personales de Aprendizaje Móvil (mPLE) en la Educación Superior (Tesis doctoral). Universidad de Salamanca, Salamanca. Recuperado de http://repositorio.grial. eu/handle/grial/635

International Telecomunications Union. (2016). UIT: Comprometida para conectar el mundo. Recuperado de http://www.itu.int:80/es/Pages/default.aspx

Lara, T. (2005). Blogs para educar. Usos de los blogs en una pedagogía constructivista. Telos: Cuadernos de comunicación e innovación, 65, 86-93.

Oviedo, H. C., \& Campo Arias, A. (2005). Aproximación al uso del coeficiente alfa de Cronbach. Revista Colombiana de Psiquiatría, XXXIV (4), 572580. Recuperado de http://www.redalyc.org/ articulo.oa?id=80634409

Torre, A. de la. (2006). Web Educativa 2.0. Edutec. Revista Electrónica de Tecnología Educativa, 0 (20). Recuperado de http://www.edutec.es/revista/ index.php/edutec-e/article/view/515

UTECA, U. (2017). Total de estudiantes legalmente matriculados en el Ciclo Académico ABRIL 2017 - AGOSTO 2017 (Reporte SICOA). Riobamba, Ecuador: Universidad Nacional de Chimborazo.

Vilhelmson, B., Thulin, E., \& Elldér, E. (2017). Where does time spent on the Internet come from? Tracing the influence of information and communications technology use on daily activities. Information Communication and Society, 20 (2), 250-263. https://doi.org/10.1080/1369118X.2016.1164741

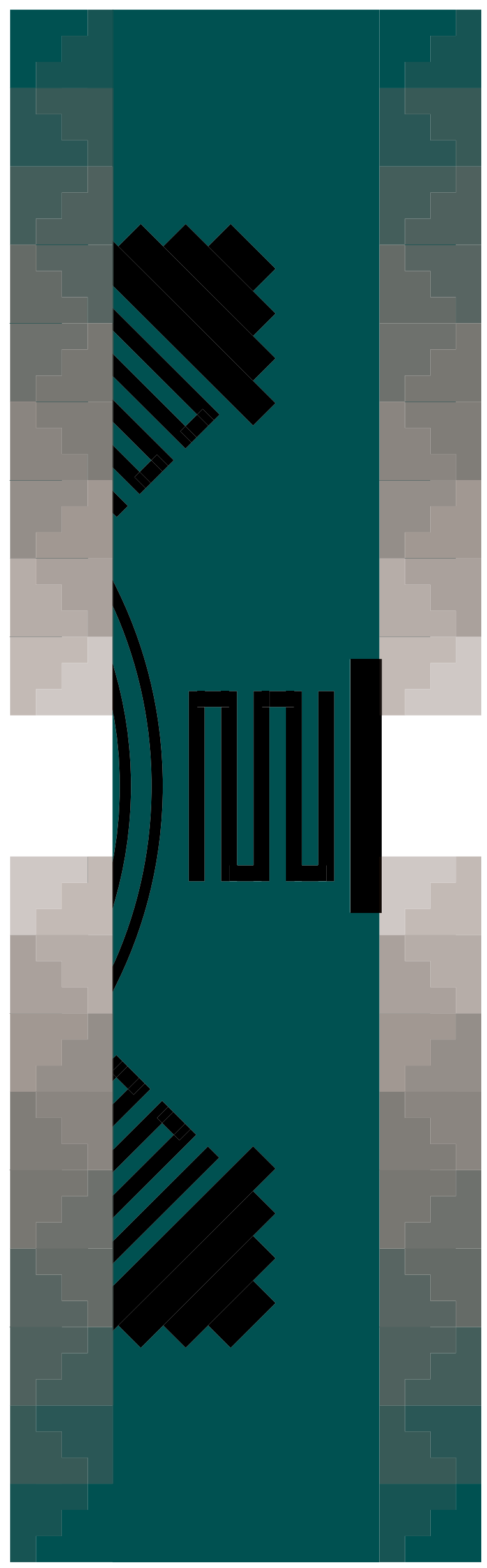

